\title{
Effects of resveratrol on bone healing capacity in the mouse tooth extraction socket
}

P-BR-059

\section{BASIC RESEARCH}

Jo-Young Suh11, Kyung Kon Min¹, Sanjiv Neupane², Nirpesh Adhikari², Wern-Joo Sohn³, Seo-Young An ${ }^{4}$,

Ji-Youn Kim ${ }^{5}$, Chang-Hyeon An ${ }^{4}$, Youngkyun Lee ${ }^{2}$, Yong-Gun Kim¹, Jin-Woo Park ${ }^{1}$,

Jae-Mok Lee ${ }^{1}$, Jae-Young Kim²

${ }^{1}$ Department of Periodontology, ${ }^{2}$ Department of Biochemistry, ${ }^{4}$ Department of Maxillofacial Surgery, School of Dentistry, IHBR, Kyungpook National University, Daegu, Korea

${ }^{3}$ Pre-Major of Cosmetics and Pharmaceutics, Daegu Haany University, Gyeongsan, Korea

${ }^{5}$ Department of Dental Hygiene, Gachon University, Incheon, Korea

\section{Abstract}

Background: After tooth extraction, the extraction socket undergoes several steps of soft and hard tissue healing. The healing process of the extraction socket is modulated by a range of signaling factors and biochemical agents. It has beer reported that resveratrol, a polyphenolic compound, exhibits various biological effects, including anti-inflammatory, anticarcinogenic, anti-oxidant, and anti-aging effects, and protect cardiovascular and bone tissues.

Aim/Hypothesis: These various effects of resveratro suggest that it may improve the wound healing of the extraction socket during the bone formation process. We examined the cellular effects of resveratrol on human periodontal ligament (hPDL) cells and the bone-healing capacity of tooth extraction socket in mice.

Material and Methods: Resveratrol $(25 \mu \mathrm{M})$ was applied to hPDL cells to detect cell proliferation and alkaline phosphatase (ALP) activity and real-time PCR was employed to understand the gene expression level in-vitro. For in-vivo experiment six weeks old $\mathrm{C} 57 \mathrm{BL} / 6$ male mice were randomly divided into control $(n=15)$ and experimental $(n=15)$ groups and maxillary first molars were extracted by surgery. Experimental group received $50 \mu \mathrm{M}$ resveratrol on extraction sockets and analyzed the degree of new bone formation.

Results: Treatment of hPDL cells with resveratrol increase the cell proliferation and ALP activity and enhanced the expression of ALP, BMP-2, BMP-4, and OC genes. Resveratro $(50 \mu \mathrm{M})$ was applied to facilitate osteogenesis and its effects were compared with those observed in the control group on days 5,7 , and 14 ; statistically significant results were obtained. No significant difference was observed in the degree of new bone formation in the apical region between the control and experimental groups, whereas bone formation was observed in the coronal region in the experimental group that was statistically significantly higher than that in the control group ( $P$ $<0.01$ ).

Conclusion: These results suggest that resveratro increases the cellular physiology of hPDL cells, including their proliferation and differentiation, and may play an important role in bone healing capacity after tooth extraction.

\section{Background and Aim}

\section{Background}

Tooth extraction is the most common and routine dental procedure. Alveolar bone resorption after tooth extraction may affect the subsequent placement of a dental implant, leading to aesthetic problems and prosthetic instability. Socket preservation technique was used to overcome the complication of insufficient bone quantity and many current studies are focusing on the development of bioactive materials that may facilitate wound healing.

Resveratrol has shown that the antioxidant and antiinflammatory properties of resveratrol inhibit the inflammatory response of periodontal tissues and prevent alveolar bone loss in rat models. In addition, resveratrol is known to stimulate the proliferation and differentiation of osteoblasts.

These various effects of resveratrol suggest that this compound may modulate the inflammation resulting from bacterial infection and traumatic extraction and improve the wound healing process of the extraction socket during the bone formation process.

\section{Aim}

In this study, we carefully evaluated the effects of resveratrol on the cellular activity of the periodontal ligament (PDL) cells, which exhibit ALP protein activity and gene expression and subsequently display the effects of the socket healing process, including new bone formation in vivo.

\section{Methods and Materials}

In vitro study

After $24 \mathrm{hr}$ and $48 \mathrm{hr}$ incubation, resveratrol was applied to hPDL cells to detect cell proliferation using CCK-8 assay and alkaline phosphatase (ALP) activity.

At day 1, 7, 14 and 21, Real-time PCR was performed to quantitate the gene expression using a specified thermal cycler with $i Q$ SYBR Green Supermix. These genes included ALP, BMP-2, BMP-4, osteocalcin and an endogenous control gene (glyceraldehyde- 3 phosphate dehydrogenase, GADPH).

\section{In vivo study}

Six-week-old $\mathrm{C} 57 \mathrm{BL} / 6$ male mice $(\mathrm{N}=30)$ were randomly divided into two groups.

(1) control ( $n=3$ for each measurement group, a total of 15 for 5 measurement groups)

(2) experimental ( $\mathrm{n}=3$ for each measurement group, a total of 15 for 5 measurement groups)

In both groups, the upper right first permanent molars were extracted by surgery. The periodontal ligament was not removed and left in extraction socket. For the control group, $2 \mu \mathrm{L}$ mixture comprising of Pluronic F-127 and $0.01 \%$ DMSO was applied on the extraction socket, while the experimental group was treated with $2 \mu \mathrm{L}$ mixture comprising of resveratrol, Pluronic F-127 and $0.01 \%$ DMSO. The final concentration of resveratrol in the mixture was maintained at $50 \mu \mathrm{M}$. The mice were sacrificed and maxillas were harvested on days 1, 3, 5, 7, and 14 after treatments.

The sections were routinely stained with hematoxylin and eosin (HE) for histological observation using a light microscope. The structural orientation and integration of PDL with bone and cementum was examined after staining with Masson's trichrome (MTC) stain. Histologic and histomorphometric evaluations were carried out.

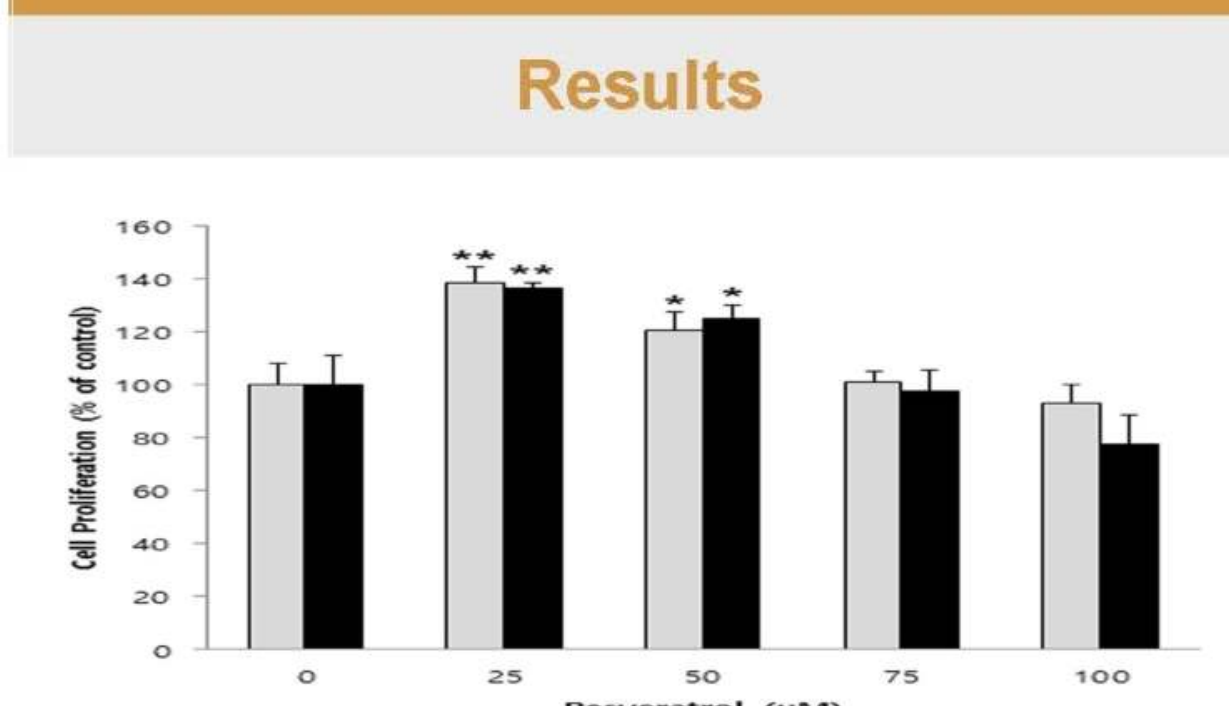

Figure 1. Effect of Resveratrol on proliferation of hPDL cell. After $24 \mathrm{hr}$ and $48 \mathrm{hr}$ incubation, cell proliferation was assessed using CCK-8 assay.

Statistically significant differences compared to control $(P<0.05)$

* : Statistically significant differences compared to control $(P<0.01)$

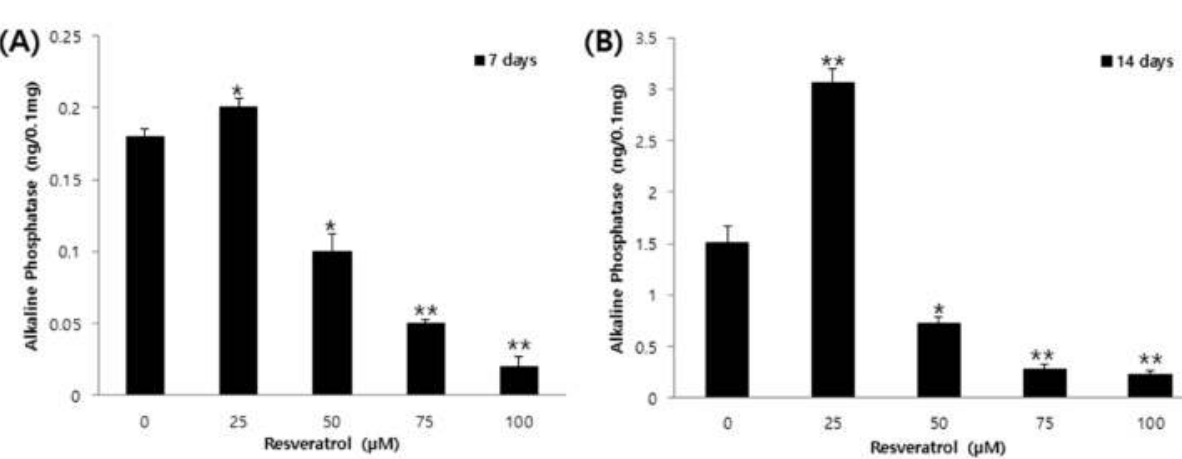

Figure 2. Alkaline Phosphatase assay. Effect of Resveratro on ALP activity in PDL cell. Cultured on days 7 and 14.
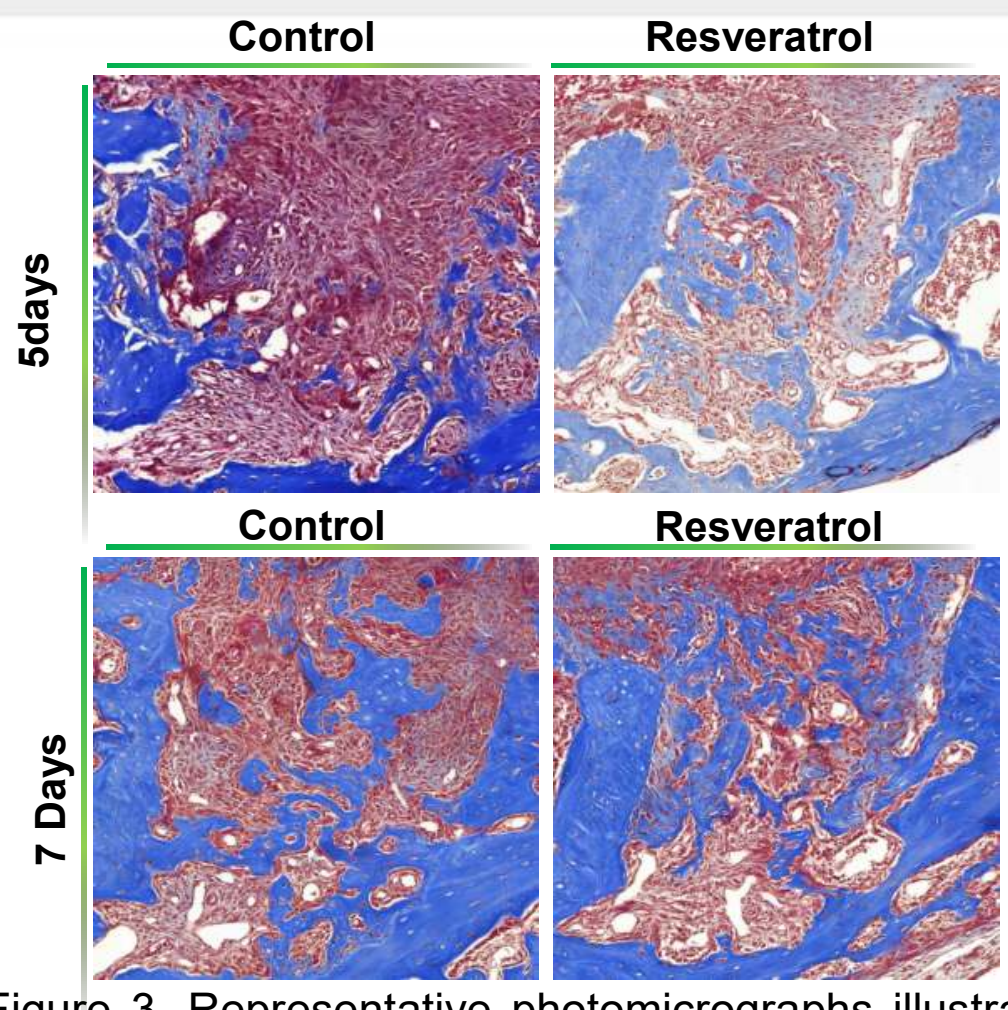

Figure 3. Representative photomicrographs illustrating the histological findings in the control group and resveratrol group at $5 \mathrm{~d}$ and $7 \mathrm{~d}$.
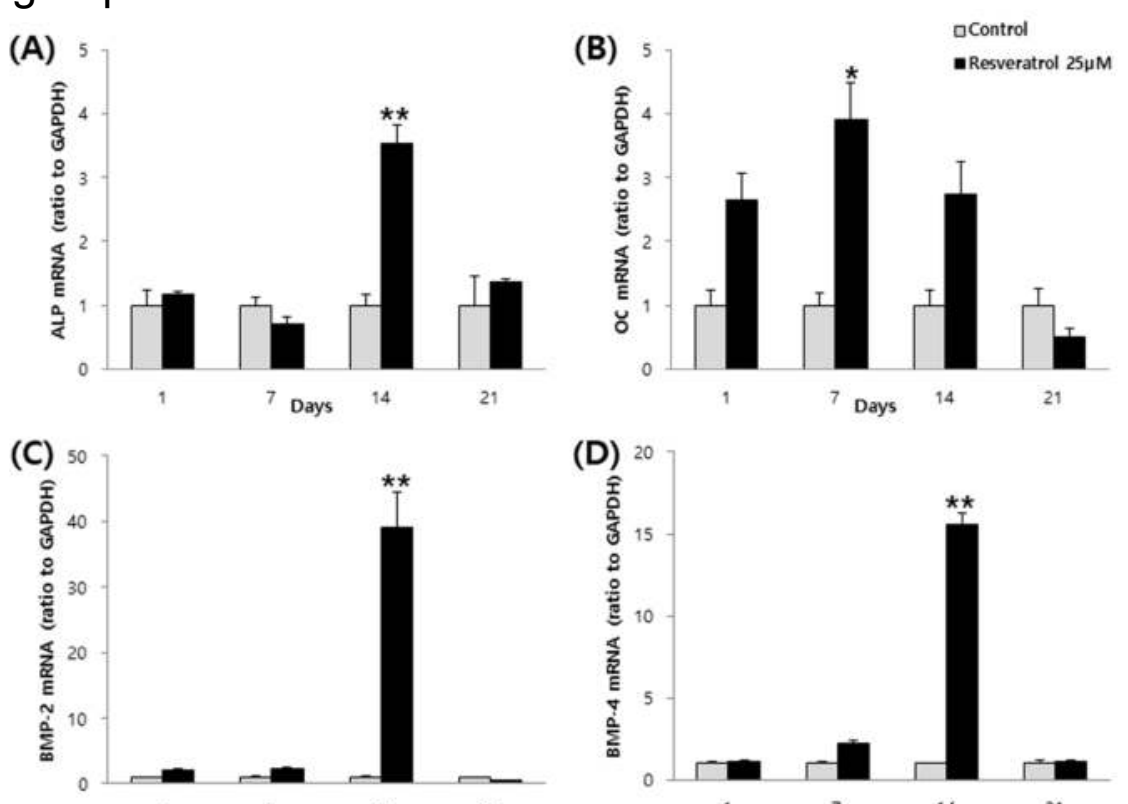

Figure 4. . Effect of resveratrol on osteogenesis-related genes (A-D) of hPDL cells. The treatment with $25 \mu \mathrm{M}$ resveratrol resulted in the highest $A L P$ expression on day 14 (A). The highest expression level of $O C$ was observed at day 7 (B). BMP-2 and BMP-4 showed high expression on day 14 and the differences were statistically significant as compared to the control group $(C, D)$.

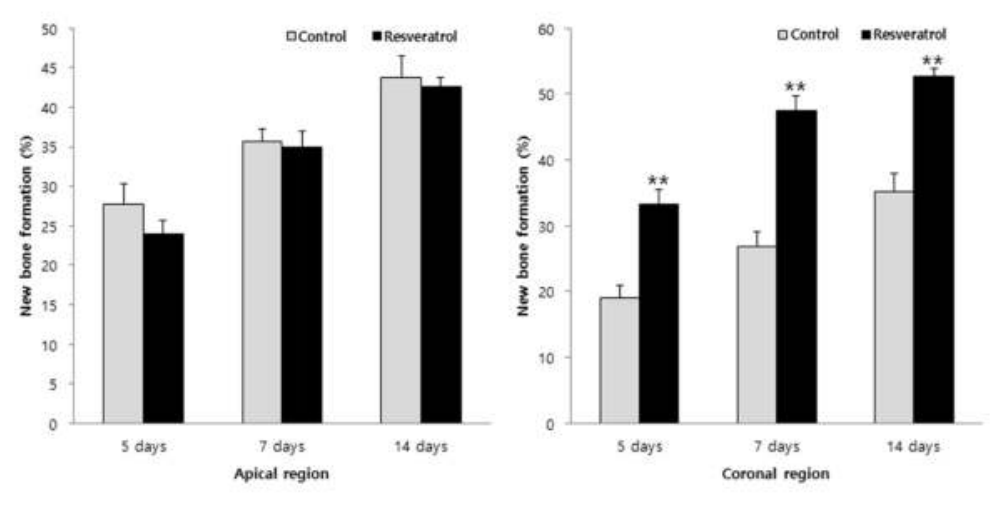

Figure 5. Effect of resveratrol on new bone formation in apical and coronal region

\section{Conclusion}

These results suggest that resveratrol increases the cellular physiology of hPDL cells, including their proliferation and differentiation, and may play an important role in bone healing capacity after tooth extraction.

\section{References}

1. Mizutani K, Ikeda K, Kawai Y, Yamori Y. Resveratrol stimulates the proliferation and differentiation of osteoblastic MC3T3-E1 cells. Biochem. Biophys. Res. Commun. 1998:253: 859-863

2. Bhattarai G, Poudel SB, K SH, Lee JC. Resveratrol prevents alveolar bone loss in an experimental rat model of periodontitis. Acta Biomaterialia 2015;29:398-408 